\title{
Noncanonical NF-kB Signaling Pathway in Liver Diseases
}

\author{
Qianhui Chen ${ }^{1}$, Xinyu $\mathrm{Lu}^{1}$ and Xiaoyong Zhang*1,2(1) \\ ${ }^{1}$ State Key Laboratory of Organ Failure Research, Guangdong Provincial Key Laboratory of Viral Hepatitis Research, \\ Department of Infectious Diseases, Nanfang Hospital, Southern Medical University, Guangzhou, Guangdong, China; \\ ${ }^{2}$ Hepatology Unit, Shenzhen Hospital, Southern Medical University, Shenzhen, Guangdong, China
}

\begin{abstract}
The noncanonical NF-KB signaling pathway is an important branch of NF-KB signaling. It is involved in regulating multiple important biological processes, including inflammation and host immune response. A central adaptor protein of the noncanonical NF-kB pathway is NF-kB-inducing kinase (NIK), which activates the downstream kinase IKKa to process p100 to $\mathrm{p} 52$, thereby forming the RelB/p52 heterodimer to initiate the expression of target genes. Currently, many specific inhibitors and monoclonal antibodies targeting or triggering this pathway are being developed and tested for various diseases, including cancers, autoimmune diseases, and virus infection. Given that aberrant activation of the noncanonical NF-KB pathway is frequently observed in various liver diseases, targeting this pathway may be a promising therapeutic strategy to alleviate liver inflammation. Moreover, activation of this pathway may contribute to the antiviral immune response and promote the clearance of persistent hepatotropic virus infection. Here, we review the role of the noncanonical NF-KB pathway in the occurrence and development of different liver diseases, and discuss the potency and application of modulating the noncanonical NF-KB pathway for treatment of these liver diseases.
\end{abstract}

Citation of this article: Chen $Q$, Lu $X$, Zhang $X$. Noncanonical NF-KB signaling pathway in liver diseases. J Clin Transl Hepatol 2021;000(000):000-000. doi: 10.14218/ JCTH.2020.00063.

\section{Introduction}

The NF- $\mathrm{kB}$ family of transcription factors, including NF- $\mathrm{kB} 1$ p50, NF-kB2 p52, RELA (p65), c-Rel and RelB, ${ }^{1}$ are involved in diverse biological processes, such as inflammation, apoptosis, proliferation, and development. ${ }^{2,3}$ These NF-KB subunits form various homodimers or heterodimers that bind to $\mathrm{KB}$ enhancers of target genes and regulate their transcription. ${ }^{3}$ In resting cells, the NF-KB dimer is inactive and is sequestered in the cytoplasm by binding to members of the $\mathrm{KB}$ inhibitory factor (IKB) family. In activating cells, NF-KB signaling is activated through a series of signaling cascades, following the ligation of various cell surface receptors with paired ligands. ${ }^{3}$

NF- $\mathrm{kB}$ signaling transduction can be divided into canonical or noncanonical NF-kB signaling pathways (Fig. 1). The canonical pathway has been well studied, and is known to rely on the degradation of IkBa. ${ }^{4}$ This pathway is also known to be rapid and transient. In contrast, activation of the noncanonical NF-KB signaling pathway has been shown to rely on the processing of $\mathrm{p} 100$. This process is characteristically slow and persistent, and is regulated in a strict and complex manner through the activity of a variety of proteins. ${ }^{5,6}$ NF- $\mathrm{kB}$-inducing kinase (NIK) is a central and specific signal component in the noncanonical NF-KB signaling pathway, 6,7 while inducible p100 processing is a central step in noncanonical NF-KB signaling transduction. ${ }^{5}$

Recent studies have suggested that the noncanonical NF$K B$ signaling pathway is involved in regulating multiple important biological processes, such as immune inflammation, development of lymphoid organs, and B and T cell survival and maturation. ${ }^{4}$ In addition, the well-characterized functions of the noncanonical NF-KB pathway are also found to be dysregulated in the pathogenesis of various liver diseases, including metabolic liver disease, autoimmune liver disease, and viral hepatitis. Here, we review the expression and function of the noncanonical NF-KB signaling molecules in various liver diseases. In particular, we also discuss the therapeutic potency of modulating noncanonical NF-KB signaling for treatment of liver diseases.

Signaling molecules of noncanonical NF-кB signaling pathway

Important signaling molecules of the noncanonical NF-KB signaling pathway include receptors and adaptor proteins. The best known noncanonical NF-KB receptors belong to the tumor necrosis factor receptor (TNFR) superfamily and include lymphotoxin beta receptor (LTBR), ${ }^{8}$ B-cell-activating factor belonging to TNF family receptor (BAFFR), 9,10 $\mathrm{CD} 40,9,10$ receptor activator for nuclear factor KB (RANK), ${ }^{11}$ 


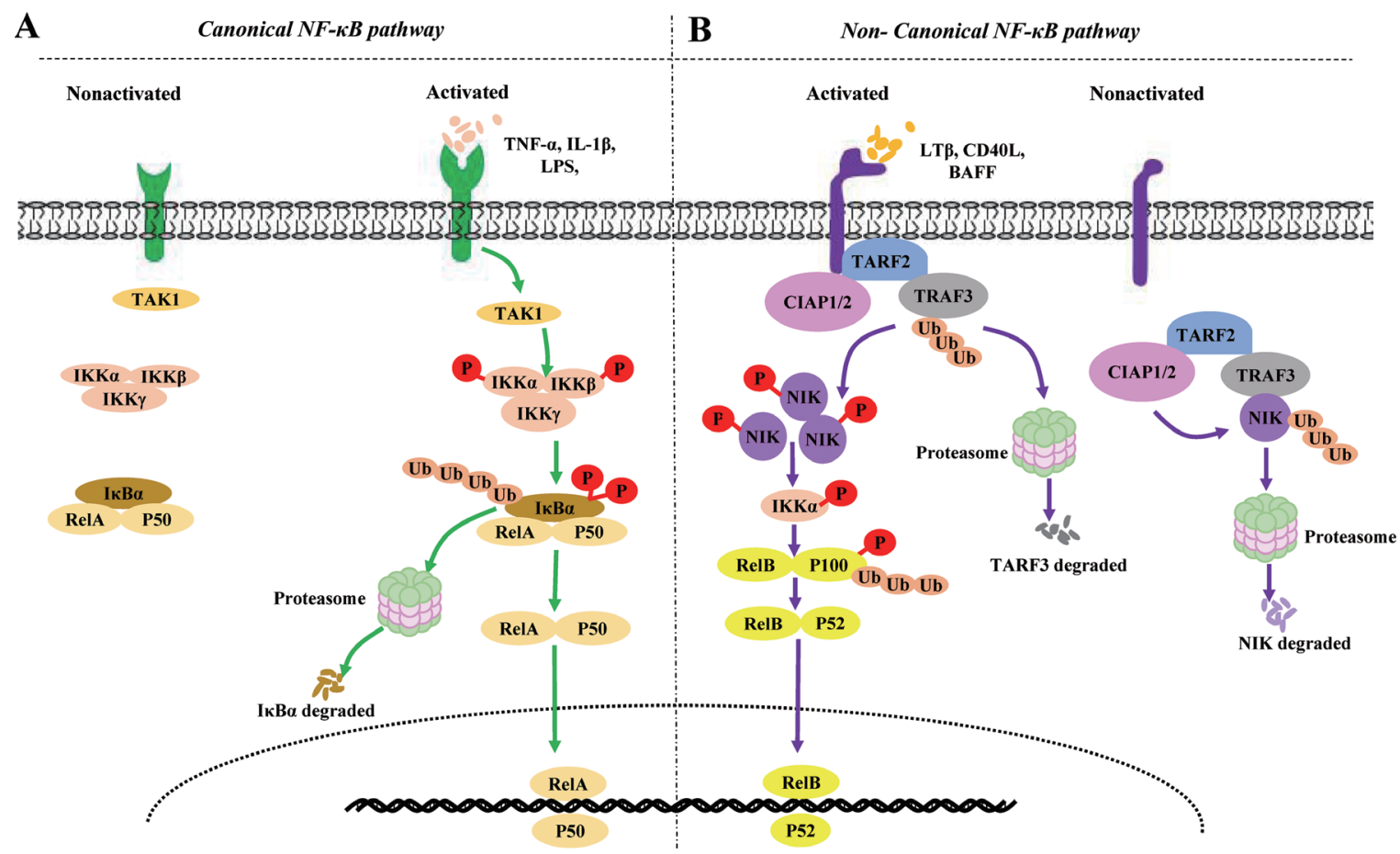

Fig. 1. Canonical and noncanonical NF-kB signaling pathways in resting vs. receptor-stimulated cells. ( $A$ ) Canonical NF- $\kappa B$ signaling pathways. In resting cells, the binding of members of the IKB family, such as the prototypical IKB member IKBa, to classical NF- $k B$ complexes, particularly NF- $k B 1$ p50-RelA and NF- $k B 1$ p50-c-Rel dimers, inhibit the nuclear translocation of NF-KB complexes. The canonical pathway is activated by signals from various immune-related receptors. In various proinflammatory cytokine-stimulated cells, ligand-receptor interactions initially activate TAK1. Subsequently, TAK1 activates the IKK complex and mediates IKBa phosphorylation and ubiquitin ( $\mathrm{Ub}$ )-dependent proteasomal degradation, leading to the rapid and transient nuclear translocation of the classical NF-KB dimers. (B) Noncanonical NF-KB signaling pathways. In resting cells, the cIAP1/2-TRAF2-TRAF3 multisubunit ubiquitin ligase complex is composed of TRAF2, TRAF3, and cIAP1/2, in which TRAF2 acts as the adaptor protein, linking TRAF3 and cIAP1/2. The binding of NIK to TRAF3 leads to the constitutive degradation of NIK in a Ub- and proteasomedependent manner. Following ligand (such as LTB, CD40L or BAFF) stimulation, the cIAP1/2-TRAF2-TRAF3 complex is recruited to the receptors. The focus of cIAP1/2 mediated k48 ubiquitination and proteasome-dependent protein degradation shifts from NIK to TRAF3, which induces TRAF3 degradation and NIK stabilization. Subsequently, phosphorylated NIK phosphorylates IKKa, which, in turn, phosphorylates NF-KB2 p100, resulting in the processing of p100 into p52 and the nuclear translocation of RelB-p52 heterodimers. In addition, stabilized NIK is regulated via a negative feedback loop, involving IKKa-mediated phosphorylation.

fibroblast growth factor-inducible 14 (Fn14), 12 and OX40 (also called CD134).13 A common feature of these receptors is the presence of a TNFR-associated factor (TRAF)binding motif, which recruits different TRAF molecules, particularly TRAF2 and TRAF3, to the receptor complex during ligand binding. This is a critical step, leading to degradation of TRAF molecules and activation of the noncanonical NFKB pathway by downstream signals. ${ }^{14,15}$ Several non-TNFR receptors can also mediate noncanonical NF-KB pathway activation, such as macrophage colony-stimulating factor receptor (MCSFR), a growth factor receptor that regulates macrophage differentiation and proliferation. 14,15

The essential adaptor proteins belong to TRAF2, TRAF3, NIK (also known as MAP3K14), IKB kinase a (IKKa) complex, precursor $\mathrm{p} 100$, RelB, and the p100 processed product, p52. In resting cells, TRAF2 acts as an adaptor protein connecting TRAF3 with cellular inhibitor of apoptosis protein (CIAP) 1/2 to form a TRAF3-TRAF2-cIAP1/2 multisubunit ubiquitin ligase complex. ${ }^{16}$ TRAF3 is not only an NIK binding protein but also a key adaptor protein that regulates the level of NIK. ${ }^{17}$ Although TRAF3 cannot catalyze the formation of K48-linked ubiquitin chains, it acts as a bridge between CIAP1/2 and NIK, thereby allowing CIAP1/2 to mediate K48 ubiquitylation and proteasome-dependent NIK degradation, which maintains NIK at a low level.5,18 When the receptor is stimulated, the target of CIAP1/2-mediated $\mathrm{K} 48$ ubiquitination and proteosome-dependent protein degradation changes from NIK to TRAF3 through raising the TRAF3-TRAF2-CIAP1/2 complex to receptors, which results in TRAF3 degradation and stabilizes NIK expression. 5,16 However, some stimulatory signals can also stabilize NIK expression by inducing the degradation of TRAF2 or CIAP. ${ }^{19-}$ 21 The accumulation of NIK induces phosphorylation and polyubiquitination of $\mathrm{p} 100$, leading to the release of $\mathrm{p} 52$. Subsequently, the RelB/p52 heterodimer translocates into the nucleus and initiates the expression of target genes ${ }^{19-21}$ (Fig. 1). The targets regulated by this pathway include several cytokine and chemokine coding genes, such as CCL19 (also called ELC), CCL21 (also known as SLC), CXCL13 (also named BLC), VCAM1, ICAM1, and MADCAM1.19-21 Inducible p100 processing by activating NIK is the centerpiece in the activation of the noncanonical NF- $\mathrm{KB}$ signaling pathway and involves the strict regulation of multiple processes, such as phosphorylation, ubiquitination, and ubiquitin-like modification. ${ }^{5}$ Notably, although IKKa is thought to be a key regulator of p100 phosphorylation, activating IKKa alone is not enough to activate the noncanonical NF-KB pathway, which additionally requires the activity of NIK.6,22

The noncanonical NF-KB signaling pathway is regulated at multiple levels. NIK, as the first central regulatory component of this pathway, is itself regulated by various factors. TRAF2, TRAF3, and CIAP are negative regulators of NIK ${ }^{19-21}$ and are essential for maintaining a low NIK level in resting cells. NIK is also regulated through a feedback mechanism involving its downstream kinase, IKKa. ${ }^{23}$ In mouse B cells and fibroblasts, TRAF3 degradation following receptor activation does not lead to a sustained increase in NIK levels but instead maintains NIK at a steady level. Furthermore, 
compared with wild-type cells, IKKa-deficient cells express higher basal levels of NIK. ${ }^{23}$ However, the negative feedback regulation of NIK by IKKa cannot replace the function of the TRAF3-TRAF2-cIAP1/2 complex, which controls the basal level of NIK.

Several additional factors have recently been implicated in the regulation of NIK. In BAFFR- and CD40-activated B cells, TANK binding kinase 1 (TBK1) phosphorylates NIK and initiates NIK degradation. ${ }^{24}$ Monarch-1 (also known as NLRP12) interacts with NIK to induce NIK ubiquitination and subsequent proteasome-dependent degradation in myeloid cells. Moreover, monarch-1 may indirectly regulate the level of NIK by stabilizing the TRAF3 level. ${ }^{25,26}$ Another potential negative regulator of NIK is TRAF- and NIK-associated protein (TNAP). In TNAP-transfected cells, the kinase activity of NIK is suppressed, which inhibits p100 processing. ${ }^{27}$ Notably, most studies investigating the role of these factors in NIK regulation have used specific cell lines, and whether their function has tissue-cell specificity merits further investigation. IKKa is also regulated by several factors. A recent study identified microRNA (miR)-223, miR-15a, and miR-16 as specific negative regulators of IKKa that regulate the activation of the noncanonical NF-KB signaling pathway during macrophage differentiation. ${ }^{28}$

\section{Role of noncanonical NF-KB signaling pathways in dif- ferent liver diseases}

The liver is an important metabolic organ of the body, and is involved in biosynthesis, biotransformation, and detoxification. In vivo, the liver is associated with the metabolism of glucose, lipids, and proteins, as well as that of alcohol, drugs, and poisons. Consequently, the liver is often affected by a wide variety of pathogenic factors. Liver damage is always closely associated with the occurrence of intrahepatic inflammation and an impaired immune response. The noncanonical NF-KB signaling pathway plays significant roles in the development and regulation of the immune system, and also has a role in multiple immune inflammatory diseases. Recent studies have demonstrated that dysregulation of the noncanonical NF-KB signaling pathway is related to the pathophysiological mechanisms associated with several liver diseases, including nonalcoholic fatty liver disease (NAFLD), alcoholic liver disease (ALD), autoimmune liver disease, and viral hepatitis (Table $1^{30-59}$ ). Furthermore, aberrant activation of the noncanonical NF-KB pathway in different cell types (such as hepatocytes and thymic epithelial cells) can elicit different effects on the pathogenesis of liver diseases. ${ }^{29-31}$

\section{NAFLD/nonalcoholic steatohepatitis/ALD}

NAFLD is an acquired, metabolic stress-induced liver injury, and is closely related to insulin resistance and genetic susceptibility. The most significant pathological feature of NAFLD is the existence of vesicular steatosis in the liver in the absence of alcohol and other well-defined liver injury-related factors. NAFLD is divided into two categories: non-alcoholic fatty liver and nonalcoholic steatohepatitis (NASH); importantly, NASH patients are prone to progression to liver fibrosis and cirrhosis. NAFLD/NASH is associated with multiple metabolic characteristics including obesity, diabetes, dyslipidemia, and hypertension. It has also been shown that B-cell-activating factor belonging to TNF family (BAFF) is linked to the histological severity of NASH. 60 Mechanistically, the BAFF/BAFFR signal was also found to exert a protective role in hepatic steatosis via the downregulation of the expression of steatogenesis-related genes in hepatocytes. ${ }^{32}$ RANK plasma levels and peripheral blood mononuclear cell mRNA levels were also found to be decreased in NAFLD patients. ${ }^{33}$ Similarly, the plasma OX40 level was also positively correlated with disease severity in $\mathrm{NASH}$ patients. OX40, a key regulator of innate and adaptive immunity in the liver, promoted NASH initiation and development by increasing the functions of proinflammatory monocytes, macrophages, and T cells. ${ }^{33}$ This suggests that the noncanonical NF-KB signaling pathway may have a role in the formation and development of NAFLD/NASH.

The activity of NIK and the expression of P52 (the activated form of NF-KB2) are increased in the liver, while NIK has been shown to promote glucagon responses in obese mice. Obese mice with liver-specific inhibition of NIK expression exhibited reduced glucagon responses and hepatic glucose production as well as resistance to hyperglycemia and glucose intolerance. ${ }^{35}$ In line with that study, NIK deletion in hepatocyte or immune cells suppressed liver inflammation and lipogenic programs, thereby protecting against high fat diet-induced liver steatosis. ${ }^{36}$ Conversely, NIK overexpression in mouse hepatocytes triggered liver injury, extensive liver inflammation, oxidative stress, and liver fibrosis, leading to weight loss and premature death. However, these effects were independent of noncanonical NF-KB signaling pathway activation. Instead, the mechanism was related to the overexpression of NIK in hepatocytes, which induced activated bone marrow-derived macrophages to secret proapoptotic molecules, which stimulated hepatocyte apoptosis. ${ }^{31}$ Other studies have demonstrated that liver-specific expression of the carboxyl terminus of HSC70-interacting protein inhibited the activation of the noncanonical NF-KB signaling pathway and reversed the liver injury caused by hepatocyte-specific overexpression of NIK through the promotion of NIK degradation. ${ }^{37}$ Moreover, B022, a smallmolecule NIK inhibitor, also suppressed NIK-induced liver inflammation and liver injury. 38 The results of those studies suggest that NIK-mediated activation of noncanonical NF$\mathrm{KB}$ signaling in the liver or immune cells may be a potential pathogenic factor for the occurrence of NAFLD and related metabolic disorders; however, the underlying mechanisms require further investigation.

ALD includes four pathological stages: alcoholic fatty liver, alcoholic hepatitis, alcoholic liver fibrosis, and alcoholic cirrhosis. The mRNA level of Fn14 was associated with acute mortality in alcoholic steatohepatitis. ${ }^{39}$ One study found that mice after alcohol intervention and ALD patients both showed abnormally high levels of NIK mRNA and p52 protein in the liver. ${ }^{40}$ Hepatocyte-specific NIK deletion was shown to protect mice from alcoholic steatosis by sustaining hepatic fatty acid oxidation, whereas NIK overexpression contributed to hepatic lipid accumulation with disrupted fatty acid oxidation, which promoted the occurrence of ALD. 40 This indicates that the hepatocyte-specific activation of NIK is associated with ALD. Although the noncanonical NF-KB signaling pathway is activated in the liver of ALD patients, whether it is necessary for ALD development remains to be verified.

\section{Drug-induced liver disease}

Drug metabolism and clearance are mainly accomplished in the liver through biotransformation and the bile secretion pathway. However, drugs can be transformed into toxic metabolites through the action of cytochrome p450 in the liver. These metabolites can induce lipid peroxidation, dyslipidemia, and ion pump inactivation, resulting in liver injury, liver failure, liver fibrosis, and cirrhosis. Innate and adaptive immunity both play important roles in druginduced liver injury. A recent study demonstrated that the 
Table 1. Expression and function of signaling molecules of the noncanonical signaling pathway in liver diseases

\begin{tabular}{|c|c|c|c|}
\hline Liver diseases & $\begin{array}{l}\text { Signaling } \\
\text { molecules }\end{array}$ & Expression and function & Ref \\
\hline \multirow[t]{5}{*}{$\begin{array}{l}\text { NAFLD/ } \\
\text { NASH/ALD }\end{array}$} & BAFFR and BAFF & $\begin{array}{l}\text { Severe obesity and insulin resistance are found in } \\
\text { BAFFR } /- \text { mice fed a high-fat diet; BAFF down-regulates } \\
\text { the expression of steatogenesis-related genes }\end{array}$ & 32 \\
\hline & RANK & $\begin{array}{l}\text { Decreased RANK plasma level and peripheral blood } \\
\text { mononuclear cell mRNA level in NAFLD patients }\end{array}$ & 33 \\
\hline & OX40 & $\begin{array}{l}\text { Plasma OX40 level is positively correlated with disease } \\
\text { severity in NASH patients; OX } 40 \text { deficiency decreases liver fat } \\
\text { accumulation, lobular inflammation, and focal necrosis in mice } \\
\text { with NASH by regulating both innate and adaptive immunity }\end{array}$ & 34 \\
\hline & Fn14 & Fn14 mRNA expression is correlated with acute mortality in ALD & 39 \\
\hline & NIK & $\begin{array}{l}\text { Liver-specific inhibition of NIK expression shows resistance } \\
\text { to hyperglycemia and glucose intolerance in obese mice; } \\
\text { NIK deletion in hepatocytes or immune cells suppress liver } \\
\text { inflammation and lipogenic programs in mice on high-fat } \\
\text { diet; Hepatocyte-specific NIK deletion protects mice from } \\
\text { alcoholic steatosis by sustaining hepatic fatty acid oxidation }\end{array}$ & $31,35-37,40$ \\
\hline \multirow[t]{2}{*}{$\begin{array}{l}\text { Drug-induced } \\
\text { liver disease }\end{array}$} & OX40 & $\begin{array}{l}\text { OX40 exacerbates paracetamol-induced liver injury by promoting } \\
\text { the function of proinflammatory macrophage and CD4+ } \mathrm{T} \text { cells }\end{array}$ & 41 \\
\hline & NIK & $\begin{array}{l}\text { Inhibiting NIK signaling ameliorates inflammation, oxidative } \\
\text { stress and damage in carbon tetrachloride-induced liver injury }\end{array}$ & 38,42 \\
\hline \multirow{5}{*}{$\begin{array}{l}\text { Autoimmune } \\
\text { liver disease }\end{array}$} & LT $\beta$ & Increased level of LT $\beta$ in the bile duct cells of PSC patients & 43 \\
\hline & BAFF & $\begin{array}{l}\text { Increased level of BAFF in peripheral blood of PBC patients; } \\
\text { BAFF promotes Treg apoptosis and inhibits cytokine } \\
\text { production by activating B cells in PBC patients }\end{array}$ & 44 \\
\hline & NIK & 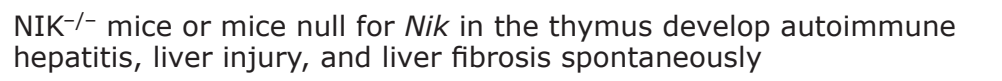 & 44 \\
\hline & IKKa & $\begin{array}{l}\text { Specific ablation of IKKa in mTECs results in severe T cell- } \\
\text { mediated autoimmune hepatitis, liver injury, and liver fibrosis }\end{array}$ & 30 \\
\hline & RelB & $\begin{array}{l}\text { Deletion of RELB from liver parenchymal cells } \\
\text { protects mice from 5-diethoxycarbonyl-1,4- } \\
\text { dihydrocollidine-induced cholestatic liver fibrosis }\end{array}$ & 43 \\
\hline \multirow[t]{5}{*}{ Viral hepatitis } & LTBR & $\begin{array}{l}\text { LTBR activation induces APOBEC3B expression, which } \\
\text { can degrade covalently closed circular DNA (the } \\
\text { template of HBV replication) in hepatocytes }\end{array}$ & 48 \\
\hline & BAFF & Increased plasma level of BAFF in patients with chronic HBV infection & 46 \\
\hline & OX40 and OX40L & $\begin{array}{l}\text { Tregs may suppress natural killer cell-mediated hepatocytotoxicity } \\
\text { directly through the interaction between OX } 40 \text { and OX } 40 \mathrm{~L} \text {, } \\
\text { which may be one of the mechanisms underlying the chronic } \\
\text { hepatitis B-associated liver disease; Treatment with OX40 } \\
\text { agonists improves HBV antigen clearance in young mice }\end{array}$ & 45,46 \\
\hline & NIK & $\begin{array}{l}\text { Overexpression of NIK stimulating by interferon-a inhibits } \\
\text { HCV replication in infected hepatocytes; Activation of NF- } \\
\text { KB in hepatocytes is occasioned by the up-regulation of NIK } \\
\text { during the initial stage of HBV infection; NIK deletion can } \\
\text { cause increased resistance to HCV infection in hepatocytes }\end{array}$ & $47,49,50$ \\
\hline & IKKa & $\begin{array}{l}\text { IKKa deletion can lead to an increase in resistance } \\
\text { to HCV infection in hepatocytes }\end{array}$ & 50 \\
\hline \multirow[t]{3}{*}{$\begin{array}{l}\text { Virus-associated } \\
\text { HCC }\end{array}$} & LTBR and LT $\beta$ & $\begin{array}{l}\text { Increased levels of } L T a \beta \text { and LTBR in HBV- or HCV- } \\
\text { induced hepatitis and HCC patients; Liver-specific LTaß } \\
\text { expression in mice induces liver inflammation and HCC }\end{array}$ & 51 \\
\hline & BAFFR & $\begin{array}{l}\text { Decreased BAFFR expression in B cells is associated } \\
\text { with tumor size and clinical stage }\end{array}$ & 52 \\
\hline & NIK & $\begin{array}{l}\text { Increased activation of NIK-dependent NF- } \\
\text { KB signal promote growth and resistance to the } \\
\text { chemotherapeutic drug 5-FU in HBV-related HCC }\end{array}$ & $53-55$ \\
\hline
\end{tabular}


Table 1. Expression and function of signaling molecules of the noncanonical signaling pathway in liver diseases - (continued)

\begin{tabular}{|c|c|c|c|}
\hline Liver diseases & $\begin{array}{l}\text { Signaling } \\
\text { molecules }\end{array}$ & Expression and function & Ref \\
\hline \multirow[t]{5}{*}{$\begin{array}{l}\text { Liver } \\
\text { regeneration }\end{array}$} & LTBR & $\begin{array}{l}\text { Mice deficient for LTBR display liver injury, low survival rates, } \\
\text { and reduction in hepatocyte proliferative capacity after PHx }\end{array}$ & 56 \\
\hline & BAFF & $\begin{array}{l}\text { The increased level of BAFF in the liver of C57/B6 mice after } \\
\text { PHx; Mice treated with anti-BAFF neutralizing antibodies } \\
\text { show impaired liver regeneration within } 72 \mathrm{~h} \text { of } 70 \% \mathrm{PHx}\end{array}$ & 57 \\
\hline & Fn14 and TWEAK & $\begin{array}{l}\text { Fn14 and TWEAK KO mice, as well as wild-type mice } \\
\text { treated with anti-TWEAK antibodies, show reduction in } \\
\text { hepatocyte and cholangiocyte proliferation after PHx }\end{array}$ & 58 \\
\hline & NIK & $\begin{array}{l}\text { Hepatocyte-specific NIK KO mice display the greater ability of } \\
\text { hepatocyte proliferation and liver regeneration after the PHX }\end{array}$ & 59 \\
\hline & IKKa & $\begin{array}{l}\text { Hepatocyte-specific IKKa KO mice display the greater ability of } \\
\text { hepatocyte proliferation and liver regeneration after the PHx }\end{array}$ & 59 \\
\hline
\end{tabular}

plasma level of OX40 was significantly increased in mice treated with paracetamol or carbon tetrachloride as well as in patients presenting with drug-induced liver injury. ${ }^{41}$ Furthermore, OX40 played a key role in promoting the function of proinflammatory macrophages and $\mathrm{CD}^{+}{ }^{+} \mathrm{T}$ cells, thereby exacerbating paracetamol-induced liver injury. ${ }^{41}$ These observations indicate that OX40/OX40L-mediated activation of the noncanonical NF-KB signaling pathway may be involved in the progression of drug-induced liver injury. Moreover, the hepatic levels of NIK MRNA and p52 protein were significantly increased in mice with carbon tetrachloride-induced liver injury. ${ }^{31,38}$ The small-molecule NIK inhibitor, B022, can inhibit NIK signaling, including noncanonical NF-KB signaling pathway activation and expression of CCL2, CCL5, CXCL5, TNF-a, IL-6, and other inflammation-related genes, thereby reducing acute liver inflammation, oxidative stress, and liver injury induced by carbon tetrachloride. ${ }^{38}$ Similarly, apigenin, a flavonoid found in many plants, can mitigate liver injury by ameliorating inflammation and oxidative stress through suppressing the noncanonical NF-KB pathway. ${ }^{42}$ These results suggest that activation of the noncanonical NF-KB signaling pathway may promote the development and progression of drug-induced liver diseases. At the same time, selective small-molecule NIK inhibitor 46 (XT2) or B022 are effective at inhibiting drug-induced liver injury and liver inflammation, which shows that NIK is an attractive drug target in drug-induced liver diseases. 38,61

\section{Autoimmune liver disease}

Autoimmune hepatitis is a type of liver inflammatory injury disease resulting from autoimmune abnormalities. Genetically susceptible individuals develop autoimmune hepatitis mostly due to environmental factors. Autoimmune hepatitis is characterized by the accumulation of immune cells that can recognize autoantigens and self-attack in the liver. Elevated serum transaminase and immunoglobulin $G$ levels, positive serum autoantibodies, and moderate-to-severe interfacial hepatitis are also commonly observed in patients with autoimmune hepatitis. The noncanonical NF-kB signaling pathway, an indispensable immunoregulatory factor, plays important roles in the development and regulation of $\mathrm{B}$ cell and $\mathrm{T}$ cell-mediated immune responses. ${ }^{5}$ Abnormal $\mathrm{T}$ cell development is an important risk factor for autoimmune diseases. NIK and its downstream signal, IKKa, are central components of the noncanonical NF-KB signaling pathway. Studies have reported that Nik knockout (KO) mice developed autoimmune hepatitis, liver damage, and liver fibrosis, and displayed growth retardation and premature death (most KO mice died before 13 weeks of age). ${ }^{29}$ Other studies have demonstrated that mice null for Nik in the thymus, but not in the liver or bone marrow, developed fatal autoimmune liver disease, intrahepatic inflammation, and liver fibrosis mediated by $\mathrm{CD}^{+}{ }^{+} \mathrm{T}$ cells. ${ }^{29}$ Similarly, specific ablation of either NIK or IKKa in mouse thymic medullary epithelial cells (mTECs) resulted in severe T cell-mediated autoimmune hepatitis, injury, and fibrosis in the liver, leading to premature death. ${ }^{30}$ The reason for this phenomenon may be related to the dysregulation of the noncanonical NF-KB pathway mediated by NIK/IKKa in mTECs, which abrogates $\mathrm{MTEC}$ development and leads to the breakdown of central $T$ cell immune tolerance. These observations indicate that dysregulation of the noncanonical NF- $\mathrm{KB}$ signaling pathway in the thymus has a role in autoimmune liver disease development, and even causes death in some severe cases, which may be related to the failure of central $\mathrm{T}$ cell immune tolerance.

Primary sclerosing cholangitis (PSC), an autoimmune disease of the liver, is a chronic cholestasis syndrome characterized by extensive inflammation and fibrosis in the intrahepatic and extrahepatic biliary tract systems. The levels of LT $\beta$ and RelB are up-regulated in the bile duct cells of patients with various chronic liver diseases, including PSC. 43 Moreover, results in mice showed that RelB activation (noncanonical NF-KB subunit) induced ductular reaction, oval cell activation and the progression of biliary fibrosis. Additionally, the transformation of the secretory and proliferative phenotype in cholangiocytes was shown to be dependent on lymphotoxin $\beta$ and RelB, ${ }^{43}$ suggesting that activation of the noncanonical NF-KB pathway in cholangiocarcinoma cells may promote PSC progression. The etiology of primary biliary cirrhosis (PBC) is also related to autoimmunity. The concentration of BAFF was increased in peripheral blood of PBC patients. In addition, BAFF-activated B cells could induce Treg cell apoptosis and reduce the expression of IL-10 and TGF- $\beta$, resulting in the loss of self-tolerance. ${ }^{44}$ Therefore, BAFF-mediated noncanonical NF-KB pathway activation in $B$ cells may be involved in the occurrence of PBC.

\section{Viral hepatitis}

In chronic viral hepatitis, continuous viral replication and dysregulated host immune function are the main causes of the progressive development of the disease. The noncanonical NF-KB pathway is closely related to the differentiation, proliferation, and maturation of various immune cells. Hence, abnormal immune function in patients with chronic viral hepatitis may be associated with dysregulation of in- 
tracellular noncanonical NF-kB pathway activation.

OX40 is one of the receptors that can activate the noncanonical NF-KB signaling pathways, maintaining $T$ cell excitation, promoting the proliferation of effector T cells and memory T cells, inducing $\mathrm{T}$ cell phenotype transformation, and inhibiting regulatory $T$ cell (Treg) differentiation and activity. Interestingly, effector $\mathrm{T}$ cell depletion and Treg accumulation have been observed in chronic virus-related hepatitis. Therefore, OX40/OX40L-induced noncanonical NF-KB pathway activation may be related to the development of chronic viral hepatitis. One study reported that an increased number of hepatic Tregs accumulated along with the recovery of liver injury in natural killer cell-mediated hepatitis $B$ virus (HBV) transgenic mice with the oversensitive liver injury phenotype, triggered by a low dose of concanavalin A. 45 Further findings indicated that Tregs may directly suppress natural killer cell-mediated hepatocytotoxicity through OX40/OX40L interaction in a cell-cell contact-dependent manner, ${ }^{45}$ which may be one of the mechanisms underlying the chronic hepatitis B-associated liver disease. Publicover et al. 46 also showed that the expression of OX40L in liver innate immune cells is essential in HBV immunization. Treatment with OX40 agonists led to improved HBV antigen clearance in young mice, and also enhanced the strength of $\mathrm{T}$ cell responses in both young mice and adult mice that had been exposed to HBV when they were young and had subsequently developed a chronic HBV infection serological profile. ${ }^{46}$ However, additional studies are needed to determine whether OX40/OX40 interaction is involved in the activation of the noncanonical NF-KB pathway in these processes. BAFF is necessary for the activation of $B$ lymphocytes. Although one study demonstrated that the serum BAFF level was higher in patients with chronic HBV infection than in the normal population, 62 the role of BAFF in the disease remains unclear.

Surprisingly, during the initial stage of HBV infection, CDNA microarray and western blot analysis showed that the mRNA and protein levels of TRAF2 and NIK were upregulated in primary normal human hepatocytes. ${ }^{47}$ In addition, LTBR, a member of the TNFR superfamily, can activate NIK-mediated noncanonical NF- $\mathrm{kB}$ signaling. The activation of LTBR induced APOBEC3B expression, which can degrade covalently closed circular DNA (the template of HBV replication) in hepatocytes. ${ }^{48}$ These findings suggest that the activation of NIK exerts antiviral effects in hepatocytes. Although NIK is an indispensable component of noncanonical NF-KB signaling pathway, NIK activity is not specific to this pathway. Thus, it is not clear whether elevated intrahepatic NIK expression exerts antiviral effects by activating the noncanonical NF-KB signaling pathway or through another as yet unidentified mechanism. Notably, HBV polymerase (pol) can inhibit the activation of the noncanonical NF-KB pathway by suppressing the nuclear translocation of NF-KB subunits in hepatoma cells, thereby antagonizing host innate immune responses. ${ }^{63}$ The above results indicate that noncanonical NF-KB pathway activation is inhibited in the liver of patients with chronic HBV infection. Recently, it was found that the Smac mimetic Birinapant, which might activate the noncanonical NF-KB pathway by targeting CIAP1and CIAP2, was able to promote the apoptosis of virusinfected hepatocytes to clear persistent HBV infection. ${ }^{64,65}$

Previous studies have shown that hepatitis $\mathrm{C}$ virus (HCV) replication can be inhibited in infected hepatocytes following interferon-a-stimulated overexpression of NIK. 49 However, NIK and IKKa deletion can lead to an increase in resistance to $\mathrm{HCV}$ infection in hepatocytes. ${ }^{50} \mathrm{HCV}$ infection promoted the expression of NIK in hepatocytes at the post-transcriptional level, and then NIK directly activated IKKa to promote $\mathrm{HCV}$ replication, lipogenesis and lipid droplet formation. ${ }^{50}$ These results suggest that activation of the noncanonical NF-KB pathway is closely related to hepatocyte susceptibil- ity to HCV. These results suggest that excessive intrahepatic NIK expression not only plays an antiviral role but also increases susceptibility to hepatitis virus infection, thereby promoting disease progression. A possible explanation for this phenomenon may be that the NIK-mediated functions of the noncanonical NF-KB pathway may differ according to the disease stage. Alternatively, NIK and IKKa may induce additional signaling molecular pathways that counteract the noncanonical NF-KB pathway, thereby leading to different effects.

\section{Viral associated hepatocellular carcinoma}

The pathogenesis of hepatocellular carcinoma (HCC) is associated with a variety of etiologies, including chronic HBV and HCV infections. The development of HCC is inseparable from the immunosuppressive microenvironment. The noncanonical NF-KB pathway plays important roles in the development and regulation of the immune system and may be involved in HCC pathogenesis. One study found that the expression of LTBR and that of its ligands (LTa and LT $\beta$ ) was up-regulated in HBV- or HCV-associated HCC, while the liver-specific expression of $\mathrm{LTa} / \beta$ can induce liver inflammation and tumor formation in mice. ${ }^{51}$ The expression of BAFFR, which is closely related to $B$ cell survival and maturation, was decreased in $B$ cells in the peripheral blood of HCC patients with chronic HBV infection, and this decreased BAFFR expression was significantly correlated with tumor size and clinical stage. ${ }^{52}$ All the above-mentioned receptors and corresponding ligands can activate the noncanonical NF-KB signaling pathway, which suggests that the activation of this signaling pathway may play an important role in the initiation and development of HCC.

In addition, the overexpression of miR-98-5p markedly inhibited the proliferation, migration, and invasive ability of tumor cells and promoted cell apoptosis in HBV-related $\mathrm{HCC}$ via decreasing the protein expression of NIK. Furthermore, overexpression of miR-98-5p also significantly inhibited tumor growth and decreased the expression of NIK in a mouse xenograft tumor model. ${ }^{53} \mathrm{HBV}$ infection promoted an increase in NIK-dependent activation of NF-KB, which not only promoted the occurrence of HBV-related HCC but was also correlated with HCC resistance to the chemotherapeutic drug fluorouracil (commonly known as 5-FU). ${ }^{54,55}$ Small interfering RNA-mediated inhibition of the NIK-dependent activation of NF- $\mathrm{KB}$ reduced resistance to 5 -FU in HBV-related HCC. 55 This suggests that HBV may promote the development of HCC and its drug resistance through noncanonical pathways mediated by NIK.

\section{Role of noncanonical NF-KB signaling pathways in liver regeneration}

The liver has a powerful regenerative capacity. Following $70 \%$ partial hepatectomy $(\mathrm{PHx})$, rodents can regain normal liver mass within a week via reparative hepatocyte replication. However, when impaired, this process can exacerbate the pathogenesis of acute or chronic liver diseases. LTBR is one of the receptors that can activate the noncanonical NF-KB signaling pathway. Mice deficient for LTBR signaling displayed clear liver injury, low survival rates, and reduced hepatocyte proliferative capacity after $\mathrm{PHx}$. Similar phenomena were also observed in mice deficient for the LTBR ligand LTa after PHx, suggesting that the LTRR/LTa signaling pathway enhances hepatocyte regeneration. ${ }^{56}$ The expression of BAFF was up-regulated in the liver of C57/B6 mice after $\mathrm{PHx}$. Conversely, within $72 \mathrm{~h}$ of undergoing $70 \% \mathrm{PHx}$, mice treated with anti-BAFF neutralizing antibodies died, showing 
reduced microvascular density in their remaining liver tissue as well as impaired liver regeneration, suggesting that BAFF-mediated signaling improved hepatocyte regeneration. ${ }^{57}$ Moreover, compared with control mice, both Fn14 and TNF-like weak inducer of apoptosis (TWEAK) KO mice showed reduced hepatocyte and cholangiocyte proliferation after PHx. A similar phenomenon was also observed in wildtype mice treated with anti-TWEAK antibodies, ${ }^{58}$ which indicated that the TWEAK/Fn14 signal is essential for mouse liver regeneration after $\mathrm{PHx}$. All of the above-mentioned receptor-ligand interactions can activate the noncanonical NF-KB signaling pathway. Consequently, these results imply that activation of the non-canonical NF- $\mathrm{kB}$ signaling pathway may be associated with liver regeneration; however, the underlying mechanism needs to be further determined.

Compared with NIK flox/flox mice, hepatocyte-specific NIK $\mathrm{KO}$ and hepatocyte-specific IKKa KO mice displayed a greater ability for hepatocyte proliferation and liver regeneration after the PHx due to the stable deletion of NIK or IKKa in hepatocytes. ${ }^{59}$ Hepatotoxin treatment or high-fat diet feeding inhibited $\mathrm{PHx}$-stimulated hepatocyte replication in mice. Under the same condition, inactivation of hepatic NIK markedly enhanced hepatocyte proliferation. 59 The above findings suggest that activation of the noncanonical NF-KB signaling pathway plays important roles in hepatocyte regeneration. Mechanistically, to some extent, activated hepatic NIK/IKKa inhibited liver regeneration by suppressing the cell mitotic pathway-JAK2/STAT3 pathway. ${ }^{59}$ Therefore, targeting the NIK may also accelerate liver regeneration, which would also be of concern in acute liver failure.

\section{Therapeutic targeting of the noncanonical NF-кB signaling pathway in liver diseases}

Given that aberrant activation of the noncanonical NF-KB pathway is observed in various liver diseases, targeting this pathway may be a promising therapeutic option. For example, the anti-BAFF monoclonal antibody belimumab has been approved to be clinically administered to systemic lupus erythematosus patients. ${ }^{66}$ Increased level of BAFF in peripheral blood can also be observed in patients with autoimmune liver disease, and a study has found that BAFF promotes Treg cell apoptosis and inhibits cytokine production by activating B cells in PBC patients. 44 Therefore, BAFF antagonists might be applicable to the autoimmune liver diseases. In addition, the results showing that selective small-molecule NIK inhibitor 46 (XT2) or B022 are effective at inhibiting drug-induced liver injury and liver inflammation indicate that NIK is an attractive target in drug-induced liver diseases. ${ }^{38,61}$ It will be interesting to test these NIK inhibitors with different scaffolds for treatment in clinical trials. Furthermore, IKKa deletion can lead to increased resistance to HCV infection and ability for hepatocyte proliferation and liver regeneration.50,59 Teglarinad chloride, one of the IKKa/ IKK $\beta$ inhibitors which has passed phase 1 clinical trials for malignant melanoma, lymphoma and solid tumors, ${ }^{67}$ may be used in treatment of HCV infection and acute liver failure. Although some specific inhibitors and/or monoclonal antibodies are currently being applied to a broad range of diseases in which aberrant activation of noncanonical NF-KB signaling occurs, further studies are needed to determine whether they can be used in the treatment of liver diseases.

\section{Conclusions}

Extensive investigation has led to a deeper understanding of the signal transduction mechanisms and biological functions associated with the noncanonical NF-KB signaling pathway. Its functions are indispensable, especially for the production of a normal immune response and in inflammatory diseases. Numerous studies have also shown that abnormalities in noncanonical NF-KB signaling are closely related to the occurrence of various liver diseases, which provides a theoretical basis for the discovery of drugs targeting the noncanonical NF-KB pathway.

Despite these advances, several questions remain to be addressed. First, most studies that have investigated the noncanonical NF-kB pathway have focused on the cytoplasmic regulation of upstream kinases, such as NIK, IKKa, and NF-KB heterodimers, while little attention has been paid to the nuclear regulation of NF-KB, especially that related to RelB-p52 heterodimers. Second, although receptor binding to specific ligands can activate the noncanonical NF-KB pathway, the target genes that are regulated by different receptor-activated signaling pathways in specific cell types remains unclear. Third, although activation of the noncanonical NF-KB pathway is completely dependent on NIK, this is not the only function of this protein. Therefore, it remains to be determined whether NIK mediates the activation of additional signaling pathways that may interact with the noncanonical NF-KB pathway. Fourth, although several studies have shown that NIK-induced noncanonical NF-KB signaling is closely related to various liver diseases, it is unclear how this pathway regulates the expression of target genes involved in the development of these diseases. Moreover, although the canonical and noncanonical NF-KB pathways are known to interact, whether this interaction is associated with the promotion of liver disease, and how the two pathways regulate each other, requires further investigation. Solving these problems will contribute to exploring more specific and effective methods for treating liver diseases based on the noncanonical NF-KB pathway.

\section{Funding}

This review was partly supported by grants from the National Natural Science Foundation of China (No. 81871664) and the National Science and Technology Major Project (No. 2018ZX10301202-003).

\section{Conflict of interest}

The authors have no conflict of interests related to this publication.

\section{Author contributions}

Collected the data and drafted the manuscript ( $X Z, C Q, L X)$, made critical revision of the manuscript for important intellectual content $(X Z)$.

\section{References}

[1] Hoesel B, Schmid JA. The complexity of NF-KB signaling in inflammation and cancer. Mol Cancer 2013;12:86. doi:10.1186/1476-4598-12-86.

[2] Mulero MC, Huxford T, Ghosh G. NF-кB, IKB, and IKK: Integral components of immune system signaling. Adv Exp Med Biol 2019;1172:207-226. doi:10.1007/978-981-13-9367-9 10

[3] Meyerovich K, Ortis F, Cardozo AK. The non-canonical NF-kB pathway and its contribution to $\beta$-cell failure in diabetes. J Mol Endocrinol 2018;61:F1F6. doi:10.1530/JME-16-0183.

[4] Sun SC. The non-canonical NF-kB pathway in immunity and inflammation. Nat Rev Immunol 2017;17:545-558. doi:10.1038/nri.2017.52

[5] Sun SC. Non-canonical NF-kB signaling pathway. Cell Res 2011;21:71-85. doi:10.1038/cr.2010.177.

[6] Tilborghs S, Corthouts J, Verhoeven Y, Arias D, Rolfo C, Trinh XB, et al. The role of Nuclear Factor-kappa B signaling in human cervical cancer. Crit Rev 
Oncol Hematol 2017;120:141-150. doi:10.1016/j.critrevonc.2017.11.001.

[7] Mitchell S, Vargas ] Hoffmann A. Signaling via the NFKB system. Wiley Interdiscip Rev Syst Biol Med 2016;8:227-241. doi:10.1002/wsbm.1331.

[8] Dejardin E, Droin NM, Delhase M, Haas E, Cao Y, Makris C, et al. The lymphotoxin-beta receptor induces different patterns of gene expression via two NF-kappaB pathways. Immunity 2002;17:525-535. doi:10.1016/ s1074-7613(02)00423-5.

[9] Claudio E, Brown K, Park S, Wang H, Siebenlist U. BAFF-induced NEMOindependent processing of NF-kappa B2 in maturing B cells. Nat Immunol 2002;3:958-965. doi:10.1038/ni842.

[10] Gardam S, Brink R. Non-canonical NF-KB signaling initiated by BAFF influences $B$ cell biology at multiple junctures. Front Immunol 2014;4:509. doi:10.3389/fimmu.2013.00509.

[11] Boyce BF, Xiu Y, Li J, Xing L, Yao Z. NF-kB-mediated regulation of osteoclastogenesis. Endocrinol Metab (Seoul) 2015;30:35-44. doi:10.3803/ EnM.2015.30.1.35.

[12] Armstrong CL, Galisteo R, Brown SA, Winkles JA. TWEAK activation of the non-canonical NF-KB signaling pathway differentially regulates melanoma and prostate cancer cell invasion. Oncotarget 2016;7:81474-81492. doi:10.18632/oncotarget.13034.

[13] Xiao X, Balasubramanian S, Liu W, Chu X, Wang H, Taparowsky EJ, et al. OX40 signaling favors the induction of $T(H) 9$ cells and airway inflammation. Nat Immunol 2012;13:981-990. doi:10.1038/ni.2390.

[14] Zhang Q, Lenardo MJ, Baltimore D. 30 years of NF-KB: A blossoming of relevance to human pathobiology. Cell 2017;168:37-57. doi:10.1016/j. cell.2016.12.012.

[15] Sun SC. Controlling the fate of NIK: a central stage in noncanonical NF-kappaB signaling. Sci Signal 2010;3:pe18. doi:10.1126/scisignal.3123pe18.

[16] Zarnegar BJ, Wang Y, Mahoney DJ, Dempsey PW, Cheung $\mathrm{HH}, \mathrm{He}$ J, et al. Noncanonical NF-kappaB activation requires coordinated assembly of a regulatory complex of the adaptors CIAP1, CIAP2, TRAF2 and TRAF3 and the kinase NIK. Nat Immunol 2008;9:1371-1378. doi:10.1038/ni.1676.

[17] Sun SC. The noncanonical NF-KB pathway. Immunol Rev 2012;246:125140. doi:10.1111/j.1600-065X.2011.01088.x.

[18] Vallabhapurapu S, Matsuzawa A, Zhang W, Tseng PH, Keats J], Wang $\mathrm{H}_{\text {, }}$ et al. Nonredundant and complementary functions of TRAF2 and TRAF3 in a ubiquitination cascade that activates NIK-dependent alternative NFkappaB signaling. Nat Immunol 2008;9:1364-1370. doi:10.1038/ni.1678.

[19] He JQ, Zarnegar B, Oganesyan G, Saha SK, Yamazaki S, Doyle SE, et al. Rescue of TRAF3-null mice by p100 NF-kappa B deficiency. J Exp Med 2006;203:2413-2418. doi:10.1084/jem.20061166.

[20] Xie P, Stunz LL, Larison KD, Yang B, Bishop GA. Tumor necrosis factor receptor-associated factor 3 is a critical regulator of $B$ cell homeostasis in secondary lymphoid organs. Immunity $2007 ; 27: 253-267$. doi:10.1016/j. immuni.2007.07.012.

[21] Gardam S, Sierro F, Basten A, Mackay F, Brink R. TRAF2 and TRAF3 signal adapters act cooperatively to control the maturation and survival signals delivered to B cells by the BAFF receptor. Immunity 2008;28:391-401. doi:10.1016/j.immuni.2008.01.009.

[22] Xiao G, Fong A, Sun SC. Induction of p100 processing by NF-kappaB-inducing kinase involves docking IkappaB kinase alpha (IKKalpha) to p100 and IKKalpha-mediated phosphorylation. J Biol Chem 2004;279:3009930105. doi:10.1074/jbc.M401428200.

[23] Razani B, Zarnegar B, Ytterberg AJ, Shiba T, Dempsey PW, Ware CF, et al. Negative feedback in noncanonical NF-kappaB signaling modulates NIK stability through IKKalpha-mediated phosphorylation. Sci Signal 2010;3:ra41. doi:10.1126/scisignal.2000778

[24] Jin J, Xiao Y, Chang JH, Yu J, Hu H, Starr R, et al. The kinase TBK1 controls IgA class switching by negatively regulating noncanonical NF-KB signaling. Nat Immunol 2012;13:1101-1109. doi:10.1038/ni.2423.

[25] Allen IC, Wilson JE, Schneider M, Lich JD, Roberts RA, Arthur JC, et al. NLRP12 suppresses colon inflammation and tumorigenesis through the negative regulation of noncanonical NF-KB signaling. Immunity 2012;36:742-754. doi:10.1016/j.immuni.2012.03.012.

[26] Lich JD, Williams KL, Moore CB, Arthur JC, Davis BK, Taxman DJ, et al. Monarch-1 suppresses non-canonical NF-kappaB activation and p52-dependent chemokine expression in monocytes. J Immunol 2007;178:12561260. doi:10.4049/jimmunol.178.3.1256

[27] Hu WH, Mo XM, Walters WM, Brambilla R, Bethea JR. TNAP, a novel repressor of NF-kappaB-inducing kinase, suppresses NF-kappaB activation. J Biol Chem 2004;279:35975-35983. doi:10.1074/jbc.M405699200.

[28] Li T, Morgan MJ, Choksi S, Zhang Y, Kim YS, Liu ZG. MicroRNAs modulate the noncanonical transcription factor NF-kappaB pathway by regulating expression of the kinase IKKalpha during macrophage differentiation. Nat Immunol 2010;11:799-805. doi:10.1038/ni.1918.

[29] Shen H, Sheng L, Xiong Y, Kim YH, Jiang L, Chen Z, et al. Thymic NF-kBinducing kinase regulates $C D 4^{+} \mathrm{T}$ cell-elicited liver injury and fibrosis in mice. J Hepatol 2017;67:100-109. doi:10.1016/j.jhep.2017.02.025

[30] Shen $\mathrm{H}$, Ji $Y$, Xiong $Y$, Kim H, Zhong $X$, Jin MG, et al. Medullary thymic epithelial NF-kB-inducing kinase (NIK)/IKKa pathway shapes autoimmunity and liver and lung homeostasis in mice. Proc Natl Acad Sci U S A 2019;116:19090-19097. doi:10.1073/pnas.1901056116.

[31] Shen $H$, Sheng $L$, Chen $Z$, Jiang $L$, Su H, Yin L, et al. Mouse hepatocyte overexpression of NF-kB-inducing kinase (NIK) triggers fatal macrophagedependent liver injury and fibrosis. Hepatology 2014;60:2065-2076. doi:10.1002/hep. 27348

[32] Kawasaki K, Abe M, Tada F, Tokumoto Y, Chen S, Miyake T, et al. Blockade of B-cell-activating factor signaling enhances hepatic steatosis induced by a high-fat diet and improves insulin sensitivity. Lab Invest 2013;93:311321. doi:10.1038/labinvest.2012.176.

[33] Hadinia A, Doustimotlagh AH, Goodarzi HR, Arya A, Jafarinia M. Plasma levels and gene expression of RANK in non-alcoholic fatty liver disease. Clin Lab 2020;66. doi:10.7754/Clin.Lab.2019.190913.

[34] Sun G, Jin H, Zhang C, Meng H, Zhao X, Wei D, et al. OX40 regulates both innate and adaptive immunity and promotes nonalcoholic steatohepatitis. Cell Rep 2018;25:3786-3799.e4. doi:10.1016/j.celrep.2018.12.006.

[35] Sheng L, Zhou Y, Chen Z, Ren D, Cho KW, Jiang L, et al. NF-KB-inducing kinase (NIK) promotes hyperglycemia and glucose intolerance in obesity by augmenting glucagon action. Nat Med 2012;18:943-949. doi:10.1038/ nm. 2756 .

[36] Liu Y, Sheng L, Xiong Y, Shen H, Liu Y, Rui L. Liver NF-kB-inducing kinase promotes liver steatosis and glucose counterregulation in male mice with obesity. Endocrinology 2017;158:1207-1216. doi:10.1210/en.2016-1582.

[37] Jiang B, Shen H, Chen Z, Yin L, Zan L, Rui L. Carboxyl terminus of HSC70interacting protein (CHIP) down-regulates NF-kB-inducing kinase (NIK) and suppresses NIK-induced liver injury. J Biol Chem 2015;290:1170411714. doi:10.1074/jbc.M114.635086.

[38] Ren X, Li X, Jia L, Chen D, Hou H, Rui L, et al. A small-molecule inhibitor of NF-KB-inducing kinase (NIK) protects liver from toxin-induced inflammation, oxidative stress, and injury. FASEB ] 2017;31:711-718. doi:10.1096/ fj.201600840R

[39] Karaca G, Xie G, Moylan C, Swiderska-Syn M, Guy CD, Krüger L, et al. Role of Fn14 in acute alcoholic steatohepatitis in mice. Am J Physiol Gastrointest Liver Physiol 2015;308:G325-G334. doi:10.1152/ajpgi.00429.2013.

[40] Li Y, Chen M, Zhou Y, Tang C, Zhang W, Zhong Y, et al. NIK links inflammation to hepatic steatosis by suppressing PPARa in alcoholic liver disease. Theranostics 2020;10:3579-3593. doi:10.7150/thno.40149.

[41] Zhang $\mathrm{C}$, Jin $\mathrm{H}$, Wang $\mathrm{Y}$, Li $\mathrm{C}$, Zhao $\mathrm{X}$, Li $\mathrm{Y}$, et al. Critical role of OX40 in drug-induced acute liver injury. Br J Pharmacol 2020;177:3183-3196. doi: $10.1111 / \mathrm{bph} .15041$.

[42] Yue S, Xue N, Li H, Huang B, Chen Z, Wang X. Hepatoprotective effect of apigenin against liver injury via the non-canonical NF-KB pathway in vivo and in vitro. Inflammation 2020;43:1634-1648. doi:10.1007/s10753-02001238-5.

[43] Elßner C, Goeppert B, Longerich T, Scherr AL, Stindt J, Nanduri LK, et al. Nuclear translocation of RELB is increased in diseased human liver and promotes ductular reaction and biliary fibrosis in mice. Gastroenterology 2019;156:1190-1205.e14. doi:10.1053/j.gastro.2018.11.018.

[44] Zhang B, Hu M, Zhang P, Cao H, Wang Y, Wang Z, et al. BAFF promotes regulatory T-cell apoptosis and blocks cytokine production by activating $\mathrm{B}$ cells in primary biliary cirrhosis. Braz J Med Biol Res 2013;46:433-439. doi:10.1590/1414-431X20132665.

[45] Chen Y, Sun R, Wu X, Cheng M, Wei H, Tian Z. CD4+CD25+ regulatory $T$ cells inhibit natural killer cell hepatocytotoxicity of hepatitis $B$ virus transgenic mice via membrane-bound TGF- $\beta$ and OX40. J Innate Immun 2016;8:30-42. doi:10.1159/000431150.

[46] Publicover J, Gaggar A, Jespersen JM, Halac U, Johnson AJ, Goodsell A, et al. An OX40/OX40L interaction directs successful immunity to hepatitis $B$ virus. Sci Transl Med 2018;10:eaah5766. doi:10.1126/scitransImed.aah5766.

[47] Ryu HM, Park SG, Yea SS, Jang WH, Yang YI, Jung G. Gene expression analysis of primary normal human hepatocytes infected with human hepatitis B virus. World J Gastroenterol 2006;12:4986-4995. doi:10.3748/wjg. 12.i31.4986.

[48] Lucifora J, Xia Y, Reisinger F, Zhang K, Stadler D, Cheng X, et al. Specific and nonhepatotoxic degradation of nuclear hepatitis B virus cccDNA. Science 2014;343:1221-1228. doi:10.1126/science.1243462.

[49] Schoggins JW, Wilson SJ, Panis M, Murphy MY, Jones CT, Bieniasz P, et al. A diverse range of gene products are effectors of the type I interferon antiviral response. Nature 2011;472:481-485. doi:10.1038/nature09907.

[50] Lowey B, Hertz L, Chiu S, Valdez K, Li Q, Liang TJ. Hepatitis C virus infection induces hepatic expression of NF-KB-inducing kinase and lipogenesis by downregulating miR-122. mBio 2019;10:e01617-e01619. doi:10.1128/ mBio.01617-19.

[51] Haybaeck J, Zeller N, Wolf MJ, Weber A, Wagner U, Kurrer MO, et al. A lymphotoxin-driven pathway to hepatocellular carcinoma. Cancer Cell 2009:16:295-308. doi:10.1016/j.ccr.2009.08.021.

[52] Khlaiphuengsin A, Chuaypen N, Sodsai P, Buranapraditkun S, Boonpiyathad T, Hirankarn $N$, et al. Decreased of BAFF-R expression and B cells maturation in patients with hepatitis B virus-related hepatocellular carcinoma. World J Gastroenterol 2020;26:2645-2656. doi:10.3748/wjg. V26.i20.2645.

[53] Fei X, Zhang P, Pan Y, Liu Y. MicroRNA-98-5p inhibits tumorigenesis of hepatitis $B$ virus-related hepatocellular carcinoma by targeting NF- $k B$-inducing kinase. Yonsei Med J 2020;61:460-470. doi:10.3349/ymj.2020.61.6.460.

[54] Chung C, Park SG, Park YM, Joh JW, Jung G. Interferon-gamma sensitizes hepatitis $B$ virus-expressing hepatocarcinoma cells to 5 -fluorouracil through inhibition of hepatitis B virus-mediated nuclear factor-kappaB activation. Cancer Sci 2007;98:1758-1766. doi:10.1111/j.1349-7006.2007.00591.x.

[55] Cho HA, Park IS, Kim TW, Oh YK, Yang KS, Kim JS. Suppression of hepatitis B virus-derived human hepatocellular carcinoma by NF-kappaB-inducing kinase-specific siRNA using liver-targeting liposomes. Arch Pharm Res 2009;32:1077-1086. doi:10.1007/s12272-009-1714-z.

[56] Anders RA, Subudhi SK, Wang J, Pfeffer K, Fu YX. Contribution of the lymphotoxin beta receptor to liver regeneration. J Immunol 2005;175:12951300. doi:10.4049/jimmunol.175.2.1295.

[57] Chou CH, Ho CM, Lai SL, Chen CN, Wu YM, Shun CT, et al. B-cell activating factor enhances hepatocyte-driven angiogenesis via B-cell CLL/lymphoma $10 /$ nuclear factor-kappab signaling during liver regeneration. Int J Mol Sci 2019;20:5022. doi:10.3390/ijms20205022.

[58] Karaca G, Swiderska-Syn M, Xie G, Syn WK, Krüger L, Machado MV, et al. TWEAK/Fn14 signaling is required for liver regeneration after partial hepatectomy in mice. PLoS One 2014;9:e83987. doi:10.1371/journal. 
pone. 0083987

[59] Xiong Y, Torsoni AS, Wu F, Shen H, Liu Y, Zhong X, et al. Hepatic NF-kBinducing kinase (NIK) suppresses mouse liver regeneration in acute and chronic liver diseases. Elife 2018;7:e34152. doi:10.7554/eLife.34152.

[60] Puri P, Sanyal AJ. The BAFFling problem of B cell-activating factor in nonalcoholic fatty liver disease. Hepatol Int 2013;7:309-312. doi:10.1007/ s12072-012-9418-8.

[61] Li Z, Li X, Su MB, Gao LX, Zhou YB, Yuan B, et al. Discovery of a potent and selective NF-KB-inducing kinase (NIK) inhibitor that has anti-inflammatory effects in vitro and in vivo. J Med Chem 2020;63:4388-4407. doi:10.1021/ acs.jmedchem.0c00396.

[62] Yang C, Li N, Wang Y, Zhang P, Zhu Q, Li F, et al. Serum levels of B-cell activating factor in chronic hepatitis $B$ virus infection: association with clinical diseases. J Interferon Cytokine Res 2014;34:787-794. doi:10.1089/ jir.2014.0032.
[63] Liu D, Wu A, Cui L, Hao R, Wang Y, He J, et al. Hepatitis B virus polymerase suppresses NF-KB signaling by inhibiting the activity of IKKs via interaction with Hsp90ß. PLoS One 2014;9:e91658. doi:10.1371/journal. pone.0091658

[64] Ebert G, Allison C, Preston S, Cooney J, Toe JG, Stutz MD, et al. Eliminating hepatitis B by antagonizing cellular inhibitors of apoptosis. Proc Natl Acad Sci U S A 2015;112:5803-5808. doi:10.1073/pnas.1502400112.

[65] Ebert G, Preston S, Allison C, Cooney J, Toe JG, Stutz MD, et al. Cellular inhibitor of apoptosis proteins prevent clearance of hepatitis B virus. Proc
inturation Natl Acad Sci U S A 2015;112:5797-5802. doi:10.1073/pnas.1502390112.

Natl Acad Sci U S A 2015; 112:5797-5802. doi: $10.1073 /$ pnas. 1502390112.
[66] Stohl W. Inhibition of B cell activating factor (BAFF) in the management of systemic lupus erythematosus (SLE). Expert Rev Clin Immunol 2017;13:623-633. doi:10.1080/1744666X.2017.1291343.

[67] Ramadass V, Vaiyapuri T, Tergaonkar V. Small molecule NF-kB pathway inhibitors in clinic. Int J Mol Sci 2020;21:5164. doi:10.3390/ijms21145164. 